ISSN 0103-5150

Fisioter. Mov., Curitiba, v. 25, n. 3, p. 533-540, jul./set. 2012 Licenciado sob uma Licença Creative Commons

\title{
Posicionamento corporal alterando a força muscular respiratória e o grau de obstrução em crianças asmáticas
}

\author{
Changes in postural position altering the respiratory muscle \\ strength and level of obstruction in asthmatic children
}

\author{
Kamary Coriolano Lins da Silva ${ }^{[a]}$, Tibério Cesar Queiroz de Andrade ${ }^{[a]}$, \\ Maíra Florentino Pessoa ${ }^{[b]}$, Armèle Dornelas de Andrade ${ }^{[c]}$ \\ [a] Fisioterapeutas pela Universidade Federal de Pernambuco (UFPE), Recife, PE - Brasil, e-mails: \\ kamarycoriolano@hotmail.com; tcq_andrade@hotmail.com \\ [b] Mestre em Fisioterapia pela Universidade Federal de Pernambuco (UFPE), Recife, PE - Brasil , e-mail: \\ mairapessoa@yahoo.com.br \\ [c] Professora do Departamento de Fisioterapia da Universidade Federal de Pernambuco (UFPE), Recife, PE - Brasil, e-mail: \\ armeledornelas@yahoo.com
}

\section{Resumo}

Introdução: A asma é uma desordem inflamatória crônica que, em casos graves, altera o fluxo aéreo, mesmo no período intercrise. Objetivo: Verificar, em crianças asmáticas, a influência das posturas sentada ereta e sentada inclinada para frente sobre o efeito da nebulização medicamentosa, avaliando as forças inspiratória e expiratória máximas (PI máx e PE máx) e o pico de fluxo expiratório (PFE). Método: 57 crianças, na faixa etária de 6 a 12 anos, foram distribuídas em três grupos: Grupo I, com crianças na postura sentada ereta e sentada inclinada para frente; Grupo II, com crianças na postura sentada ereta antes e após a nebulização; e Grupo III, com crianças na postura sentada inclinada para frente antes e após a nebulização. As crianças foram avaliadas três vezes nas medidas analisadas. Para comparação, foi utilizado o teste t de student (pareado) Resultados: A PI máx, a PE máx e o PFE foram maiores na posição sentada inclinada para frente, com $\mathrm{p}=0,003, \mathrm{p}=0,006$ e $\mathrm{p}=0,000$, respectivamente. Após a nebulização, o Grupo II apresentou aumentos de 44,16\% na PI máx, 36,02\% no PEF e 34,88\% na PE máx. Após a nebulização, o Grupo III apresentou elevação de 69,46\% na PI máx, 60,87\% na PE máx e 52,05\% no PFE. Comparados os grupos II e III, os ganhos são maiores no Grupo III, com $(p<0,05)$ para as medidas do PFE e da PE máx. Conclusão: A postura sentada 
inclinada para frente oferece maior vantagem mecânica para a musculatura expiratória, favorecendo a redução da obstrução, por evidenciar aumentos na PE máx e no PFE.

Palavras-chave: Asma. Força muscular respiratória. Posicionamento corporal.

\section{Abstract}

Introduction: Asthma is a chronic inflammatory disorder which in severe cases changes the air flow, even in the period between episodes. Objective: To evaluate, in asthmatic children under the effect of nebulized drug, the influence of upright sitting and leaning forward sitting postures, by assessing the intensity of the inspiratory and expiratory pressures (MIP and MEP) and peak expiratory flow (PEF). Methods: 57 children aged 6-12 years were divided in three groups: In Group I, both postures, upright sitting and leaning forward sitting, were assessed without using nebulized drug; in Group II, only the upright sitting posture was assessed before and after nebulization; in Group III, only the leaning forward sitting posture was assessed before and after nebulization. The measures were repeated by assessing these children three times in a single session. A student $t$-test (pared) was used for comparisons. Results: MIP, MEP and PEF were higher in the leaning forward position indicating respective $p$ values of $p=0.003, p=0.006$ and $p=0.000$. After nebulization, the Group II demonstrated an increase of $44.16 \%$ in MIP, 36.02\% in PEF and 34.88\% in MEP. After nebulization, the Group III showed a gain of $69.46 \%$ in MIP, 60.87 in MEP and $52.05 \%$ in PEF. The difference between groups II and III were significant $(p<0.05)$ for levels of PEF and MEP, indicating that the Group III showed better performance during these tests. Conclusion: It can be suggested that the leaning forward sitting posture provided an increased mechanical advantage for expiratory muscles, favoring the airflow during expiration and reducing the obstruction as evidenced by MEP and PEF increase.

Keywords: Asthma. Respiratory muscle strength. Body position.

\section{Introdução}

A asma é uma desordem inflamatória crônica das vias aéreas que provoca hiper-responsividade brônquica $(1,2)$. Em situações de asma moderada a grave, as alterações da luz brônquica persistem em grau reduzido, mesmo no período intercrise (3). Para reversão da obstrução, os agentes de primeira linha no tratamento são os $\beta_{2}$-agonistas adrenérgicos e os anticolinérgicos sob a forma de aerossol $(4,5)$, depositando medicação nos tecidos-alvo, maximizando a eficácia e reduzindo os efeitos colaterais sistêmicos (6). Uma falha na terapia frequentemente pode ser explicada por uma pobre entrega da medicação ao sítio de ação (7), o que poderia ser otimizado utilizando-se estratégias que modifiquem a dinâmica respiratória, como o uso de padrões ventilatórios e posturas.

0 aumento da resistência expiratória e a redução do recolhimento elástico pulmonar $(8,9)$ promovem alteração dos volumes pulmonares: há aumento do volume residual (VR) e da capacidade residual funcional (CRF) e diminuição da capacidade vital (CV) e do volume expiratório forçado no primeiro segundo $\left(\mathrm{VEF}_{1}\right)$, num fenômeno conhecido como "Síndrome da Hiperinsuflação" $(10,11)$.

Essa Síndrome da Hiperinsuflação altera a conformação e a função dos músculos respiratórios, levando-os a desvantagens mecânicas $(7,12,13)$. A redução da pressão transdiafragmática torna-o deficiente gerador de pressão $(3,7,8)$, prejudicando a troca gasosa e ativando os quimiorreceptores, que recrutam os acessórios da respiração, aumentando a CRF (5-7). A utilização dos acessórios com fins respiratórios pode resultar em fadiga muscular e aumento da sensação de dispneia $(14,15)$.

Alguns estudos $(16,17)$ analisaram a relação entre posturas corporais, a atividade dos músculos respiratórios e sua desvantagem mecânica. As mudanças de posturas poderiam influenciar, principalmente, na posição do músculo diafragma, no aumento da tensão relacionada a outros músculos, tais como os abdominais, e no efeito da gravidade sobre eles.

0 presente estudo visa correlacionar o efeito das posturas sentada inclinada para frente e sentada ereta e suas principais repercussões durante a avaliação 
da força muscular respiratória, com e sem o uso da broncodilatação, através do Pico de Fluxo Expiratório (PFE), da Pressão Inspiratória Máxima (PI máx) e da Pressão Expiratória Máxima (Pe máx) em crianças asmáticas.

\section{Casuística e método}

\section{Amostra}

Constituída por 57 crianças, 25 do sexo masculino e 32 do sexo feminino, com idade média de 9,02 $\pm 1,7$, diagnóstico médico de asma brônquica e questionário de estadiamento da asma, estando no período intercrise, caracterizado pela ausência de sintomas há um mês, e cujos responsáveis assinaram o termo de assentimento. Foram triadas e avaliadas no serviço de alergologia do Hospital das Clínicas da Universidade Federal de Pernambuco (UFPE) e no serviço de fisioterapia da Central de Alergologia do Estado de Pernambuco. 0 projeto foi aprovado no Comitê de Ética e Pesquisa da UFPE, de acordo com a Resolução 196/96 CNS.

\section{Desenho do estudo}

Foi um estudo randomizado e cego, dividido em três grupos experimentais (Figura 1):

- Grupo I ( $\mathrm{n}=15)$ : crianças avaliadas na posição sentada ereta e sentada com o tronco inclinado para frente e os cotovelos apoiados nos joelhos (tronco inclinado a $45^{\circ}$ ), comparando PI máx, PE máx e PFE nas duas posturas;

- Grupo II ( $\mathrm{n}=23)$ : crianças avaliadas comparando PI máx, PE máx e PFE antes e após a nebulização com tronco ereto, usando broncodilatadores numa solução de beta-adrenérgico de curta duração - Fenoterol (2,5 mg) - e um anticolinérgico - Ipratrópio $(0,25 \mathrm{mg})$-, somados a solução salina a $0,9 \%$, completando $5 \mathrm{ml}$ (solução padrão).

- Grupo III ( $\mathrm{n}=19)$ : crianças avaliadas comparando PI máx, PE máx e PFE antes e após a nebulização com tronco inclinado 45oㅡ, usando broncodilatadores na solução padrão.

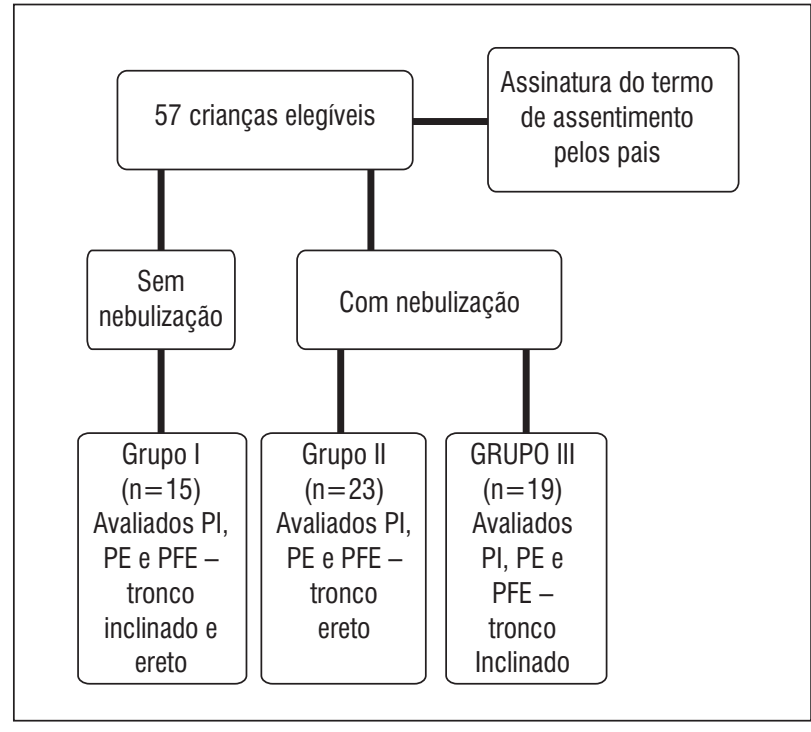

Figura 1 - Fluxograma da aleatorização Fonte: Dados da pesquisa.

No Grupo I, o intuito foi avaliar os efeitos da postura sobre as variáveis do estudo (PFE, PI máx e PE máx). Nos grupos II e III, observou-se a resposta desses parâmetros avaliados antes e após o uso dos broncodilatadores, variando a postura da nebulização nas posições sentada ereta e sentada inclinada para frente.

\section{Procedimento experimental}

Avaliou-se a PI máx no manovacuômetro MV-150 (Marshal Town Instrumentation), nas respectivas posturas, com a orientação de inspirar da capacidade residual funcional (CRF) até a capacidade pulmonar total (CPT). Na PE máx, o indivíduo expirava da CRF até o volume residual (VR). Em ambas as situações, utilizou-se uma boquilha de $5 \mathrm{~cm}$, com orifício lateral de $2 \mathrm{~mm}$ de diâmetro para escape aéreo, reduzindo a ação dos músculos bucinadores (18).

O PFE foi avaliado nas duas posturas, com um peack flow Asthec, com a orientação de expirar todo o ar contido nos pulmões de uma só vez, a partir da CPT, indo até o VR. Todas as aferições foram realizadas três vezes, com um clipe nasal, sendo capturado o melhor dos três resultados.

A posição de tronco inclinado para frente foi obtida com o indivíduo apoiando os cotovelos nas coxas (Figura 2) e mensurada por meio de um goniômetro (marca Carci), tendo como ponto móvel a linha 


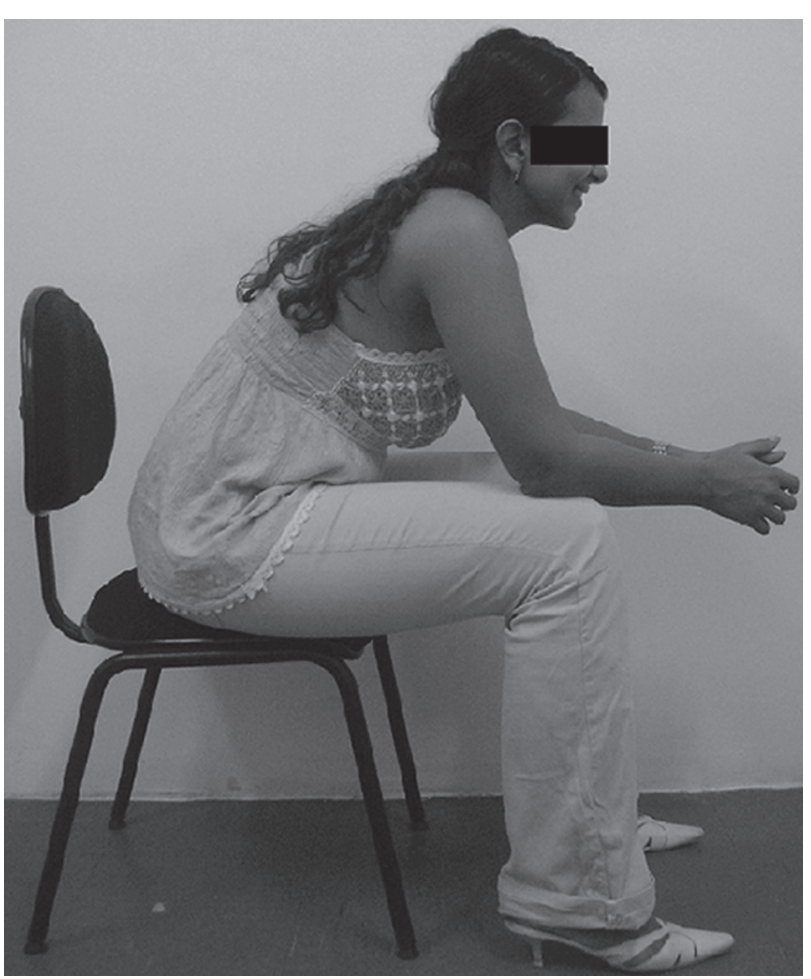

Figura 2 - Simulação da postura sentada com 0 tronco inclinado a $45^{\circ}$

Fonte: Dados da pesquisa.

axilar anterior e como ponto fixo o côndilo lateral do fêmur. 0 tempo de nebulização era o necessário para que todo o volume da solução fosse ofertado ao indivíduo, numa média de dez minutos, sendo as nebulizações instituídas pelo pesquisador 01 , com os dados aferidos pelo pesquisador 02 , quinze minutos a partir do término da aerossolterapia.

\section{Análise estatística}

Utilizou-se o software SPSS, versão 15.0. Para análise estatística, foi usado o teste $t$-student de comparações pareadas intragrupo e, para comparação nas posturas ereta e inclinada (grupos II e III), foi realizado o teste $t$-student de comparação de médias para amostras independentes, utilizando um intervalo de confiança de $95 \%$.

\section{Resultados}

A amostra foi considerada normal em todos os grupos, para os valores aferidos previamente. A média de idade do Grupo I foi de 8,67 $\pm 1,7$ anos. As médias na posição de tronco inclinado resultam em valores maiores que as da posição sentada ereta. Os resultados do teste t-student de comparações pareadas, apresentado na Tabela 2, mostram que as diferenças das médias nas duas posições analisadas são significativas, confirmando que a posição inclinada para frente favorece a mecânica respiratória (Tabela 1).

Os dados da Tabela 1 revelam ainda que os ganhos relativo da posição inclinada são maiores na PE máx $(127,84$ ou $27,84 \%)$, seguindo da PI máx $(120,65$ ou $20,65 \%)$ e do PFE $(111,70$ ou $11,70 \%)$.

Os pacientes dos grupos submetidos a nebulização (II e III) apresentam idade média de 9,13 \pm 1,6 anos, tendo as medidas após a nebulização sempre maiores que os valores prévios, em ambos os grupos. Observase que tanto na postura sentada ereta quanto na de tronco inclinado proporciona-se maiores ganhos na aferição da PI máx.

Quanto à postura sentada ereta, podemos afirmar que há um ganho em ordem decrescente para a PI máx $(144,16$ ou $44,16 \%)$,), seguida do PFE $(136,02$ ou $36,02 \%)$, finalizando com a PE máx $(134,88$ ou $34,88 \%)$. Já na postura sentada com o tronco inclinado, os ganhos afetam em ordem decrescente a PI máx $(169,46$ ou $69,46 \%)$, a PE máx $(160,87$ ou $60,87 \%)$ e o PFE $(152,05$ ou $52,05 \%)$.

Os resultados comprovam haver diferença significativa nos ganhos entre os grupos II e III (Tabela 3), no PFE e na PE máx, definidos pelo valor de p, e, embora a PI máx tenha obtido maior média de ganho do Grupo III, seus valores não atingiram níveis significantes (Gráfico 1).

\section{Discussão}

A pressão máxima e o pico de fluxo expiratório foram medidos em duas posturas, a fim de determinar a influência da postura na musculatura respiratória. Foi usada a nebulização para broncodilatação e observado o efeito das posições corporais: sentada ereta e sentada inclinada para frente, comparando-as com o antes e o após a nebulização. A pressão máxima é um indicador da força muscular respiratória, assim como o PFE registra os níveis de obstrução. Ambos têm sido usados em várias pesquisas e comparados a outros parâmetros, como idade e sexo (19).

Estudos mostram a mudança da função respiratória com a postura de pé e sentada, fazendo 
Tabela 1 - Medidas descritivas das variáveis dos pacientes do Grupo I $(n=15)$

\begin{tabular}{lccc}
\hline Variáveis & PFE & PI máx & PE máx \\
\hline Tronco ereto & $204,1 \pm 55,5$ & $53,7 \pm 11,7$ & $49,3 \pm 17,7$ \\
Tronco inclinado & $228,0 \pm 63,0$ & $64,8 \pm 19,0$ & $63,1 \pm 20,9$ \\
Ganho relativo (\%) tronco inclinado/ereto & 111,70 & 120,65 & 127,84 \\
T valor & $-3,566$ & $-3,237$ & $-3,785$ \\
P valor & $0,003^{*}$ & $0,006^{*}$ & $0,000^{*}$ \\
\hline
\end{tabular}

Fonte: Dados da pesquisa.

Legenda: ${ }^{*} p<0,05$.

Nota: Valores expressos em média \pm desvio padrão.

Tabela 2 - Medidas descritivas das variáveis dos pacientes dos grupos II e III

\begin{tabular}{lcccccc}
\hline & \multicolumn{3}{c}{ Tronco ereto $(\mathbf{n}=\mathbf{2 3 )}$} & \multicolumn{3}{c}{ Tronco inclinado $(\mathbf{n}=\mathbf{1 9})$} \\
\hline \multicolumn{1}{c}{ Variáveis } & PFE & PI máx & PE máx & PFE & PI máx & PE máx \\
\hline Pré-nebulização & $152,2 \pm 33,0$ & $46,4 \pm 17,8$ & $46,8 \pm 15,7$ & $167,2 \pm 43,8$ & $50,3 \pm 13,9$ & $53,6 \pm 13,4$ \\
Pós-nebulização & $207,1 \pm 44,0$ & $66,8 \pm 19,4$ & $63,1 \pm 18,2$ & $254,3 \pm 40,8$ & $85,2 \pm 24,8$ & $86,2 \pm 13,4$ \\
Ganho relativo (\%) & 136,02 & 144,16 & 134,83 & 152,05 & 169,46 & 160,87 \\
pré/pós-nebulização & & $-7,970$ & $-7,071$ & $-9,630$ & $-6,870$ & $-7,701$ \\
T valor & $-9,713$ & $0,000^{*}$ & $0,000^{*}$ & $0,000^{*}$ & $0,000^{*}$ & $0,000^{*}$ \\
P valor & $0,000^{*}$ & & & & & \\
\hline
\end{tabular}

Fonte: Dados da pesquisa.

Legenda: ${ }^{*} p<0,05$.

Nota: Valores expressos em média desvio \pm padrão.

Tabela 3 - Comparação de médias da \% de ganho pós-nebulização, entre os grupos II e III

\begin{tabular}{lcccc}
\hline & & PFE & PI máx & PE máx \\
\hline \multirow{2}{*}{ Teste estatístico: } & valor de t & $-2,523$ & $-1,714$ & $-2,566$ \\
& valor de $p$ & $0,018^{*}$ & 0,094 & $0,014^{*}$ \\
\hline
\end{tabular}

Fonte: Dados da pesquisa.

Legenda: ${ }^{*} p<0,05$.

referência ao comportamento dos músculos respiratórios $(20,21)$. No entanto, várias posturas poderiam ser adotadas durante a avaliação da força respiratória e cada uma dessas posições, de pé, em decúbito dorsal, em decúbito lateral e sentada oferecem ao indivíduo uma mecânica respiratória diferente (22).

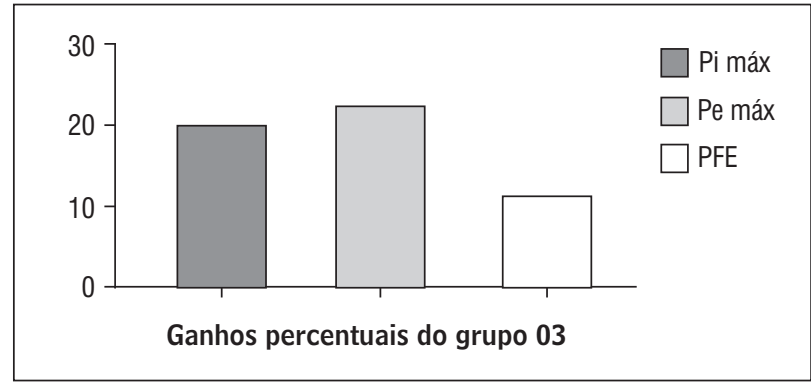

Gráfico 1 - Ganho percentual do Grupo III em relação ao Grupo II Fonte: Dados da pesquisa.

Os resultados apresentados mostram aumento da PI máx nos mesmos indivíduos, quando comparados sentados e na forma ereta ou com inclinação dianteira. Esses resultados corroboram os experimentos de Tribastone (23), que verificou que a posição de pé, com o tórax ligeiramente inclinado para frente, daria mais mobilidade às primeiras costelas, 
facilitando a elevação do diafragma provocada pela ação das vísceras. Da mesma forma, a postura sentada inclinada para frente traz, segundo Kera (21) e De Troyer (24), a mesma vantagem mecânica da musculatura respiratória se comparada à postura em pé com o tronco inclinado para frente, pois as vísceras comprimidas aumentariam a pressão intra-abdominal, empurrando o diafragma e resultando em equilíbrio da relação tensão e comprimento, o que resultaria em maior capacidade de gerar força e, por consequência, maiores valores de PI máx. Estudos de Numa (25) reforçam que a atividade diafragmática é cerca de três vezes mais intensa na postura sentada ereta do que nas posições deitada e de pé.

Entretanto, Ng e Stokes (26) discordam, afirmando que, para indivíduos no pós-operatório e os que apresentem boas condições de saúde, a PI máx e a PE máx não são significativamente diferentes quando medidas em mais de uma atitude postural. Todavia, tal disparidade nos resultados pode ser explicada por nosso estudo se propor a analisar esse desfecho nas crianças com asma de moderada a grave, no período intercrise.

Já os valores da PE máx podem ser explicados pela maior tensão gerada nos músculos abdominais, pela expansão da parede abdominal, principalmente no músculo reto do abdômen (27). A contração dos músculos abdominais, incluindo o reto e o transverso, puxa a parede abdominal dorsalmente, aumentando a pressão intra-abdominal, onde se dá o início da fase expiratória, sendo que, no começo da fase inspiratória, o reto abdominal se contrai e se distende ventralmente, diminuindo a pressão intra-abdominal. Esses movimentos influenciam o diafragma, que, associado à postura adequada, pode ter suas vantagens mecânicas aumentadas $(24,27)$.

Quanto à resposta dos broncodilatadores às posturas, foram observados resultados animadores nos grupos II e III. Durante longo tempo, os broncodilatadores ocuparam a principal posição no tratamento da asma. Com o melhor entendimento da fisiopatologia, passaram a um plano secundário, sendo utilizados atualmente apenas para o alívio das crises (28). Para este estudo, a medicação foi fundamental, pois otimiza o efeito das posturas, provocando maior deposição na musculatura lisa, melhorando o fluxo aéreo e, consequentemente, o da mecânica respiratória, favorecendo os valores percentuais para o PFE, a PE máx e o PI máx após a nebulização.
A força inspiratória elevada favorece a geração de fluxo inspiratório, que transporta mais medicamento para a via aérea (29). A otimização da força expiratória promovida pela inclinação do tronco favorece a expulsão do ar, reduzindo a hiperinsuflação dinâmica e a sensação de dispneia, permitindo a instalação de um padrão ventilatório mais lento, o que auxilia a deposição medicamentosa. Esses fatores, em conjunto, aumentam o PFE, pois elevam a força expiratória e reduzem a obstrução.

Durante a crise asmática, é possível observar o mau funcionamento da musculatura ventilatória, denotada por uso dos acessórios da respiração e pela presença de tiragens. 0 trabalho inadequado resulta em deficit ventilatório, que induz taquipneia, podendo resultar em fadiga $(29,30)$. Daí a importância de uma postura que não só promova gradualmente uma vantagem mecânica respiratória ao asmático, mas, também, possibilite boa resposta após a nebulização.

\section{Considerações finais}

Os resultados obtidos mostram que a dinâmica respiratória pode ser influenciada pela posição corporal e pelo efeito dos broncodilatadores, indicando que na postura sentada inclinada para frente o diafragma trabalha em vantagem mecânica, quando comparado a outras posturas corporais. Assim, este trabalho evidencia que a postura sentada inclinada para frente é a mais indicada para a administração da terapêutica inalatória, percebendo os efeitos nos músculos respiratórios e no grau de obstrução brônquica, além de sugestionar a escolha da postura mais adequada para outros recursos fisioterapêuticos.

Além disso, os resultados abrem perspectivas para novos estudos, visando analisar a deposição pulmonar das drogas quando nebulizadas em diferentes posturas e as repercussões de alterações posturais na mecânica respiratória.

\section{Referências}

1. Miller MG, Weiler JM, Baker R, Collins J, D’Alonzo G. Position statement: management of asthma in athletes. J Athl Train. 2005;40(3):224-45. PMid:16284647. 
2. Silva TLP, Sampaio LMM, Borghi-Silva A, Kunikoshita LM, Costa D. Comparação entre o teste de caminhada de seis minutos realizado no corredor e na esteira rolante em mulheres asmáticas. Fisioter Mov. 2007;20(2):137-44.

3. Leemans J, Kirschvink N, Bernaerts F, Clercx C, Cambier C, Gustin P.A pilot study comparing the antispasmodic effects of inhaled salmeterol, salbutamol and ipratropium bromide using different aerosol devices on muscarinic bronchoconstriction in healthy cats. Vet J. 2009;180(2):236-45. doi:10.1016/j.tvjl.2007.11.008.

4. Asher MI, Keil U, Anderson HR, Beasley R, Crane J, Martinez F, et al. International study of asthma and allergies in childhood (ISAAC): rationale and methods. Eur Respir J. 1995;8:483-91. doi:10.1183/09031936.95.08030483.

5. Nickel C, Kettler C, Muehlbacher M, Lahmann C,Tritt $\mathrm{K}$, Fartacek R, et al. Effect of progressive muscle relaxation in adolescent female bronchial asthma patients: a randomized, double-blind, controlled study. J Psychosom Res. 2005;59(6):393-8. doi:10.1016/j. jpsychores.2005.04.008.

6. Wort SJ. The management of acute severe asthma in adults. Am J Respir Crit Care Med. 2003;14(2):81-9.

7. Dolovich MB, Ahrens RC, Hess DR, Anderson P, Dhand $\mathrm{R}$, Rau JL, et al. Device selection and outcomes of aerosol therapy: evidence-based guidelines. Chest. 2005;127(1):335-71. doi:10.1378/chest.127.1.335.

8. Dalcin PTR, Perin C. Management of acute asthma in adults in the emergency room: current evidence. Rev Assoc Med Bras. 2009;55(1):82-8. doi:10.1590/ S0104-42302009000100021.

9. Nishimura Y, Tsutsumi M, Nakata H, Tsunenari T, Maeda $\mathrm{H}$, Yokoyama M. The effect of aging on respiratory muscle function. Nihon Kyobu Shikkan Gakkai Zasshi. 1991;29(7):795-801.

10. Gil FR, Lauzón AM. Smooth muscle molecular mechanics in airway hyperresponsiveness and asthma. Can J Physiol Pharmacol. 2007;85(1):133-40. doi:10.1139/y06-096.

11. Omachi TA, Iribarren C, Sarkar U, Tolstykh I, Yelin EH, Blanc P, et al. Risk factors for death in adults with severe asthma. Ann Allergy Asthma Immunol. 2008;101(2):1306. doi:10.1016/S1081-1206(10)60200-1.

12. Guha A. Transport and deposition of particles in turbulent and laminar flow. Annu Rev Fluid Mech. 2008;40:311-41. doi:10.1146/annurev.fluid.40.111406.102220.

13. Kwong KYC, Morphew T, Scott L, Guterman J, Jones CA. Asthma control and future asthma-related morbidity in inner-city asthmatic children. J Allergy Clin Immunol. 2008;101(2):144-52. doi:10.1378/chest.104.4.1108.
14. Christensen EF, Norregaard O, Jensen LW, Dahl R. Inhaled Beta $_{2}$-Agonist and positive expiratory pressure in bronchial asthma: influence on airway resistance and functional residual capacity. Chest.1993;104:1108-13. doi:10.1378/chest.104.4.1108.

15. Clifton IJ, Fletcher LA, Beggs CB, Denton M, Peckham DG. A laminar flow model of aerosol survival of epidemic and non-epidemic strains of Pseudomonas aeruginosa isolated from people with cystic fibrosis. BMC Microbiol. 2008;8:105-15. doi:10.1186/1471-2180-8-105.

16. Kera T, Maruyama H. Study of influence factor on maximal mouth pressure part I - influence of posture. J Phys Ther Sci. 2001;13:153-60. doi:10.1589/jpts.13.153.

17. Kera T, Maruyama H. Study of influence factor on maximal mouth pressure part II - influence of trunk flexion. J Phys Ther Sci. 2001;13:161-6. doi:10.1589/ jpts.13.161.

18. Hakala K, Stenius-Aarniala B, Sovijärvi A. The effect of weight loss on peack flow variability, airwaiys obstruction, and lugs volumes in obese patients with asthma. Chest. 2000;118(5):1315-21. doi:10.1378/ chest.118.5.1315.

19. Pereira CA, Sato T, Rodrigues SC. New reference values for forced spirometry in white adults in Brazil. J Bras Pneumol. 2007;33(4):397-406. doi:10.1590/ S1806-37132007000400008.

20. Decramer M. Hyperinflation and respiratory muscle interaction. Eur Respir J. 1997;10(4):934-41. PMid:9150337.

21. Kera T, Maruyama $H$. The effect of posture on respiratory activity of the abdominal muscles. J Physiol Anthropol Appl Human Sci. 2005;24(4):259-65. doi:10.2114/ jpa.24.259.

22. Crosbie WJ, Myles S. An investigation into the effect of postural modification on some aspects of normal pulmonary function. Physiotherapy. 1985;71(7):311-14.

23. Tribastone, F. Tratado de exercício corretivo aplicado à reeducação motora postural. São Paulo: Manole; 2001.

24. De Troyer A. Mechanical role of the abdominal muscles in relation to posture. Respir Physiol. 1983;53(3):341-53. doi:10.1016/0034-5687(83)90124-X.

25. Numa AH, Hammer J, Newth CJ. Effect of prone and supine positions on functional residual capacity, oxigenation, and respiratory mechanics in ventilated infants and children. Am J Respir Crit Care Med. 1997;156(4 Pt 1):1185-9. PMid:9351620. 
26. Ng GY, Stokes MJ. Maximal inspiratory and expiratory mouth pressures in sitting and half-lying positions in normal subjects. Respir Med. 1991;85(3):209-11. doi:10.1016/S0954-6111(06)80081-8.

27. Inbar O, Naiss S, Neuman E, Daskalovich J. The effect of body posture on exercise and hyperventilation - induced asthma. Chest. 1991;100(5):1229-34. doi:10.1378/ chest.100.5.1229.

28. Ninane V, Rypens F, Yernault JC, de Troyer A. Abdominal muscle use during breathing in patients with chronic airflow obstruction. Am Rev Respir Dis. 1992;146(1):1621. PMid:1385684.
29. Ogiwara S, Miyachi T. Effect of posture on ventilatory muscles strength. Phys Ther Sci. 2002;14:1-5. doi:10.1589/jpts.14.1.

30. Stell IM, Polkey MI, Rees J, Green M, Moxhan J. Inspiratory muscle strength in acute asthma. Chest. 2001;120(3):1230-36. doi:10.1378/chest.120.3.757.

Recebido: 26/04/2011

Received: 04/26/2011

Aprovado: 06/03/2012

Approved: 03/06/2012 